\title{
Morphing Low-Affinity Ligands into High-Avidity Nanoparticles by Thermally Triggered Self-Assembly of a Genetically Encoded Polymer
}

\author{
Andrew J. Simnick, ${ }^{\dagger}$ C. Alexander Valencia, ${ }^{\ddagger}$ Rihe Liu, $^{\ddagger}$ and Ashutosh Chilkoti ${ }^{\dagger, *}$ \\ ${ }^{\dagger}$ Department of Biomedical Engineering, Duke University, Durham, North Carolina 27708 and ₹School of Pharmacy and Carolina Center for Genome Sciences, University of \\ North Carolina, Chapel Hill, North Carolina 27599
}

$\mathrm{T}$ argeted drug delivery, first proposed in 1906, is a strategy for preferentially increasing the concentration of a drug at a target site relative to healthy tissue. An important goal in targeted therapy, hence, is to design a drug carrier that has high affinity and selectivity for the site of disease but exhibits low affinity for, and low interaction with, healthy tissue. Although high-affinity vehicles can show greater accumulation at the site of disease as compared to normal tissues, ${ }^{2}$ high affinity can also result in reduced specificity ${ }^{3}$ and increased toxicity ${ }^{4}$ because of "off-site" targeting to healthy tissue that also expresses the same receptor, albeit at lower levels. To circumvent this fundamental paradox, we hypothesized that an ideal targeted delivery system should have a low affinity for its target in healthy tissue but transform into a high-avidity construct at the site of disease via an extrinsic trigger (e.g., a physical stimulus such as the focused application of heat, light, or magnetic fields). We term this approach, in which a molecule morphs from a low-affinity to a high-avidity state in response to an external stimulus, dynamic affinity modulation (DAM).

Our design of a system that is capable of exhibiting DAM focused on triggered self-assembly. Multivalency, the simultaneous interaction of multiple ligandreceptor pairs, is described by

$$
K^{\text {multi }}=\left(K^{\text {mono }}\right)^{\alpha N}
$$

where $K_{\text {multi }}$ is the effective multivalent affinity (avidity), $K_{\text {mono }}$ is the affinity of a single receptor-ligand interaction, $\alpha$ is the degree of cooperativity, and $N$ is the number
ABSTRACT Multivalency is the increase in avidity resulting from the simultaneous interaction of multiple ligands with multiple receptors. This phenomenon, seen in antibody - antigen and virus - cell membrane interactions, is useful in designing bioinspired materials for targeted delivery of drugs or imaging agents. While increased avidity offered by multivalent targeting is attractive, it can also promote nonspecific receptor interaction in nontarget tissues, reducing the effectiveness of multivalent targeting. Here, we present a thermal targeting strategy — dynamic affinity modulation (DAM) — using elastin-like polypeptide diblock copolymers $\left(\mathrm{EL}_{\mathrm{B}} \mathrm{S} S\right)$ that self-assemble from a low-affinity to high-avidity state by a tunable thermal "switch", thereby restricting activity to the desired site of action. We used an in vitro cell binding assay to investigate the effect of the thermally triggered self-assembly of these $\mathrm{ELP}_{\mathrm{B} C} \mathrm{~S}$ on their receptor-mediated binding and cellular uptake. The data presented herein show that (1) ligand presentation does not disrupt $\operatorname{EL}_{\mathrm{BC}}$ self-assembly; (2) both multivalent ligand presentation and upregulated receptor expression are needed for receptor-mediated interaction; (3) increased size of the hydrophobic segment of the block copolymer promotes multivalent interaction with membrane receptors, potentially due to changes in the nanoscale architecture of the micelle; and (4) nanoscale presentation of the ligand is important, as presentation of the ligand by micrometer-sized aggregates of an ELP showed a low level of binding/uptake by receptor-positive cells compared to its presentation on the corona of a micelle. These data validate the concept of thermally triggered DAM and provide rational design parameters for future applications of this technology for targeted drug delivery.

KEYWORDS: block copolymer - polypeptide - multivalency - self-assembly · ligand-receptor

of ligand-receptor pairs. ${ }^{5}$ Thus, multivalency increases in avidity proportional to the number of simultaneous ligand-receptor interactions. Although conventional, "static" multivalent targeting is an emerging approach for targeted delivery, it suffers from the same problem of offsite targeting as monovalent, high-affinity delivery systems. ${ }^{6}$

We hypothesized that multivalency via triggered self-assembly would permit the design of a targeted delivery system exhibiting DAM. In order to design a system that could self-assemble into a multivalent construct in response to an external stimulus (Figure 1A), we focused our attention on

\section{*Address correspondence to chilkoti@duke.edu.}

Received for review November 30, 2009 and accepted March 15, 2010.

Published online March 24, 2010. $10.1021 / \mathrm{nn} 901732 \mathrm{~h}$

(c) 2010 American Chemical Society 


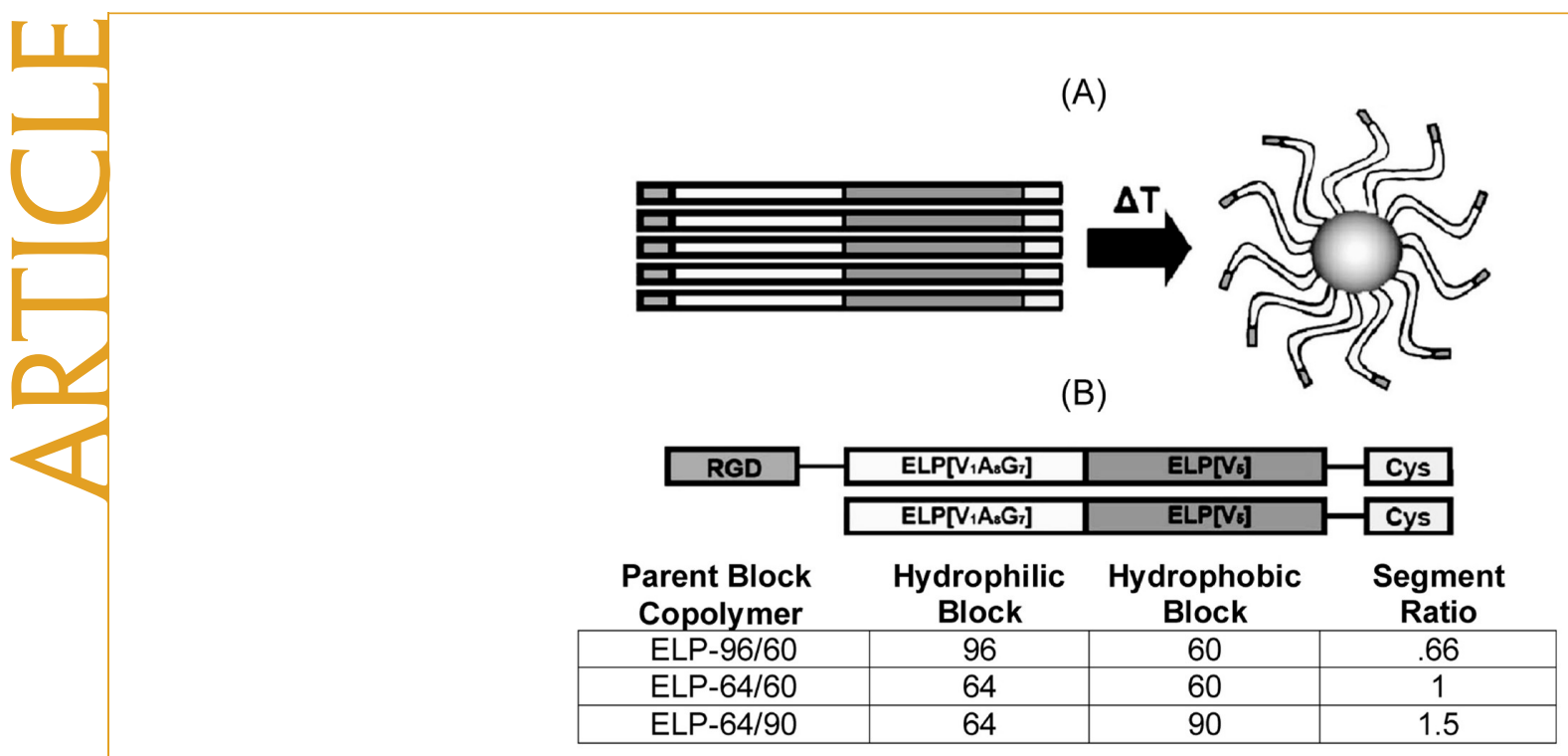

(C)
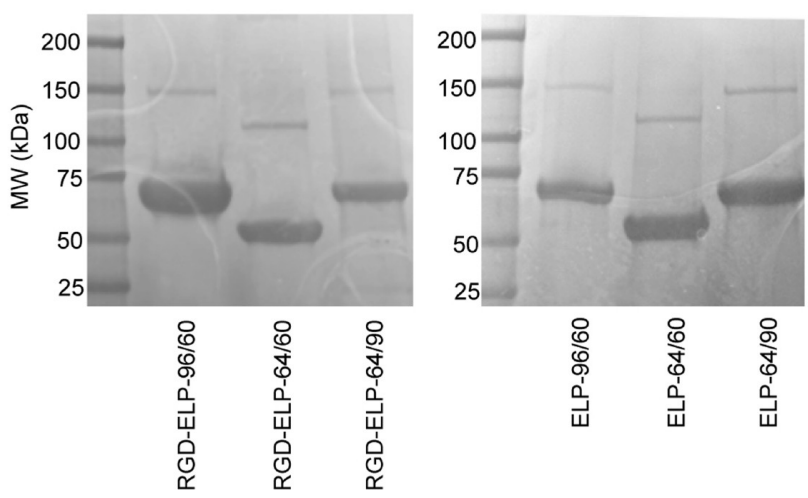

Figure 1. (A) Schema of DAM via temperature-triggered self-assembly of an ELP $\mathrm{B}_{\mathrm{BC}}$. At $T<\mathrm{CMT}$, ELP $\mathrm{B}_{\mathrm{BC}} \mathrm{S}$ exist as soluble unimers and lead to monovalent ligand presentation. At $T>C M T$, the $E \mathrm{P}_{\mathrm{BC}}$ unimers self-assemble into micelles following desolvation and collapse of the hydrophobic block. This leads to multivalent ligand presentation in the corona of the micelle. (B) ELP $_{\mathrm{BC}} \mathrm{S}$ incorporate an RGD peptide ligand at the hydrophilic terminus and a cysteine residue for conjugation of fluorophores (or drugs) at the hydrophobic terminus. The ligand-negative, control ELP $P_{B C}$ does not contain the terminal RGD ligand but includes the C-terminal cysteine. (C) SDS-PAGE of purified RGD-ELP $\mathrm{BC}_{\mathrm{BC}} \mathrm{S}$ (left) and parent ELP $\mathrm{BC}_{\mathrm{BC}} \mathrm{S}$ (right) yields a thick band corresponding to monodisperse purified protein, showing that $\mathrm{ELP}_{\mathrm{BC}}$ can be purified by ITC.

a class of diblock, stimulus-responsive elastin-like polypeptides (ELPS). ELPs are genetically encoded polypeptides composed of a Val-Pro-Gly-Xaa-Gly repeat (Xaa = any amino acid besides Pro) that exhibit inverse phase transition behavior at a specific transition temperature $\left(T_{\mathrm{t}}\right)$; ELPs are soluble in water at $T<T_{\mathrm{t}}$ and become insoluble at $T>T_{\mathrm{t}}{ }^{7-9}$ We chose diblock ELP block copolymers $\left(E \mathrm{P}_{\mathrm{BC}} \mathrm{S}\right)$ to create a system capable of DAM for several reasons. First, we and others have previously shown that $\operatorname{ELP}_{\mathrm{BC}} \mathrm{S}$ consisting of one hydrophilic and one hydrophobic ELP block are thermally triggered amphiphiles. The ELP $P_{B C}$ is a hydrophilic unimer that self-assembles into monodisperse spherical micelles with a diameter of $\sim 40-60 \mathrm{~nm}$ above a critical micelle temperature (CMT) through selective desolvation and collapse of the hydrophobic block. ${ }^{10,11}$ These micelles are stable with increasing temperature (typically $\sim 8-10{ }^{\circ} \mathrm{C}$ beyond the (MT) up to a second transition temperature, beyond which the desolvation and collapse of the hydrophobic block leads to aggregation of the $E P_{B C}$ into polydisperse micrometer-sized aggregates. ${ }^{7}$ Second, ELP $_{\mathrm{BC}} \mathrm{S}$ are monodisperse, which provides exquisite control over their self-assembly and, consequently, the size and coordination number of the micelle. This precise control is not readily possible with synthetic polymers. ${ }^{10}$ Third, ELP $\mathrm{BC}_{\mathrm{BC}} \mathrm{S}$ can be easily expressed at high levels in E. coli and conveniently purified by inverse transition cycling (ITC), ${ }^{12}$ a method that exploits the ELP phase transition to purify them directly from cell lysate without chromatography.

We chose the linear GRGDS peptide as the ligand and the $\alpha_{v} \beta_{3}$ integrin as the target receptor to demonstrate proof-of-concept of DAM using ELP $\mathrm{BC}_{\mathrm{BC}} \mathrm{S}$ as the scaffold to present the RGD peptide ligand (Figure 1B). GRGDS is a well-known, low-affinity ligand $\left(\mathrm{IC}_{50}=1\right.$ $\mu \mathrm{M})$ for the $\alpha_{\mathrm{v}} \beta_{3}$ integrin. ${ }^{13}$ The low affinity of this ligand reduces specific binding in monovalent form ${ }^{13}$ but can exhibit higher avidity through multivalent presentation. ${ }^{5}$ First, we hypothesized that linear GRGDS is a useful ligand for DAM as the large difference in affinity 

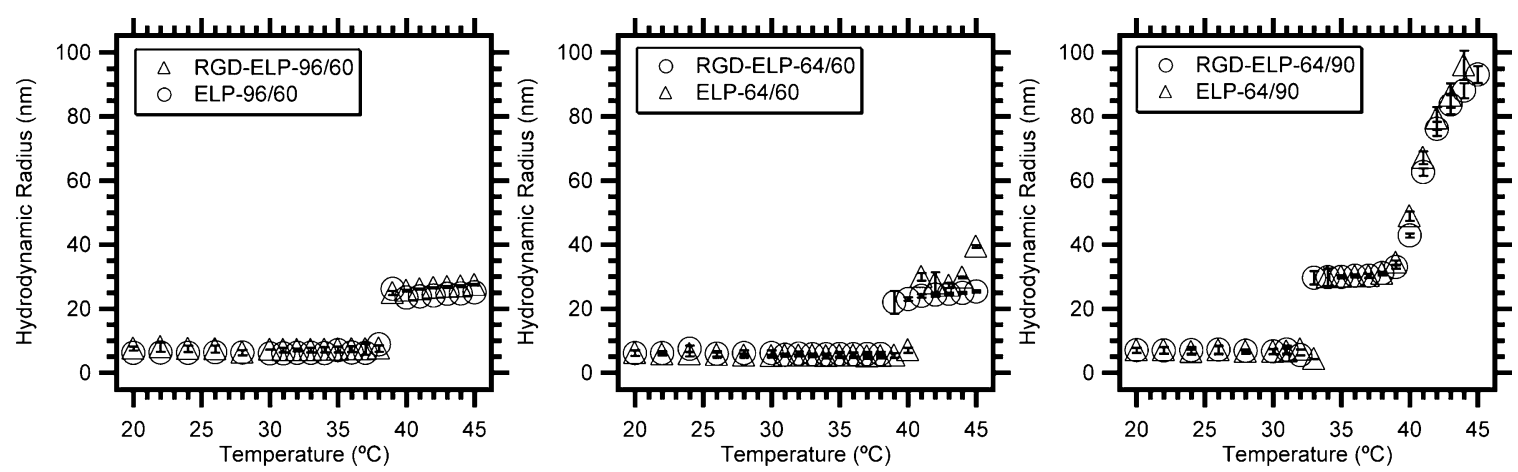

\begin{tabular}{|c|c|c|c|c|c|c|}
\hline Construct & $\mathbf{C M T}\left({ }^{\circ} \mathbf{C}\right)$ & $\begin{array}{c}\mathbf{M W}_{\text {unimer }} \\
(\mathbf{k D a})\end{array}$ & $\begin{array}{c}\mathbf{M W}_{\text {micelle }} \\
\mathbf{( k D a )}\end{array}$ & $\mathbf{Z}$ & $\mathbf{R}_{\mathbf{h}}(\mathbf{n m})$ & Termini/nm $^{\mathbf{2}}$ \\
\hline RGD-ELP-96/60 & 39 & 63.8 & 6292 & 105 & 25.0 & .0123 \\
\hline ELP-96/60 & 39 & 63.2 & 7381 & 114 & 26.7 & .0117 \\
\hline RGD-ELP-64/60 & 39 & 50.1 & 5920 & 99 & 24.3 & .0112 \\
\hline ELP-64/60 & 41 & 49.5 & 8391 & 140 & 28.3 & .0128 \\
\hline RGD-ELP-64/90 & 33 & 63.5 & 10348 & 159 & 30.9 & .0122 \\
\hline ELP-64/90 & 34 & 62.9 & 10551 & 162 & 31.8 & .0118 \\
\hline
\end{tabular}

Figure 2. Dynamic, thermally triggered self-assembly of RGD-ELP $P_{B C} S$ and $E L P_{B C} S$. Hydrodynamic radius $\left(R_{\mathrm{h}}\right)$ and molecular weight $(\mathrm{MW})$ of the $\mathrm{ELP}_{\mathrm{BC}} \mathrm{S}$ were measured as a function of temperature by DLS. Top: Both ELP $\mathrm{BC}_{\mathrm{BC}} \mathrm{S}$ exhibit distinct and stable unimer and micelle regions as a function of solution temperature. The temperature at which the unimer to micelle transition occurs is defined as the CMT (dashed line). Bottom: Presentation of the RGD peptide ligand on the hydrophilic terminus of the ELP ${ }_{B C}$ altered the self-assembly properties of the ELP-64/90 less than the ELP-96/60 or ELP-64/60 constructs. The resulting terminus density was similar for all ligand and nonligand constructs.

between its multivalent and monovalent states provides the possibility for selective receptor binding only through multivalent presentation so that this construct would localize in a target tissue that overexpresses the receptor in response to spatially focused mild hyperthermia of that tissue. Second, the $\alpha_{v} \beta_{3}$ integrin is overexpressed in angiogenic blood vessels that are associated with diseases such as cancer, ${ }^{14,15}$

atherosclerosis, ${ }^{16,17}$ and Alzheimer's disease, ${ }^{18}$ ensuring that this approach may be relevant to a number of diseases. Third, the RGD ligand is somewhat promiscuous as it binds to the $\alpha_{11 B} \beta_{3}$ integrin on activated platelets and the $\alpha_{\mathrm{v}} \beta_{3}$ integrin on healthy angiogenic tissue ${ }^{19}$ in addition to tumor vasculature. Thus, restricting RGD activity through externally triggered self-assembly would yield a benefit to this particular targeting strategy.

Fourth, the $\alpha_{v} \beta_{3}$ integrin exhibits clustering during activation, ${ }^{20}$ which should help promote the proper geometry for multivalent interaction of receptors with RGD ligands presented on an $\mathrm{ELP}_{\mathrm{BC}}$ micelle. Fifth, the linear, hydrophilic GRGDS peptide is trivial to incorporate on one of the termini of the ELP via genetically encoded synthesis ${ }^{10}$ without disrupting self-assembly of $\operatorname{ELP}_{\mathrm{BC}} \mathrm{S}$ into micelles.

This study investigates two important issues relevant to the design of a targeting system based on DAM using stimulus-responsive $\operatorname{ELP}_{\mathrm{BC}} \mathrm{S}$ as a selfassembling macromolecule and mild hyperthermia as the trigger. First, the high-avidity state of the $\operatorname{ELP}_{\mathrm{BC}}$ must be turned "on" in response to an external trigger. Sec-

www.acsnano.org ond, the size and architecture of the $\mathrm{ELP}_{\mathrm{BC}}$ micelle must facilitate multivalent interaction following ligand pre-

sentation through self-assembly. The studies presented herein attempt to address these issues by examining, in detail, the effects of both multivalency and nanoscale architecture on the interaction of a set of $\operatorname{ELP}_{\mathrm{BC}} \mathrm{S}$ with a target membrane-based receptor. We chose three selfassembling diblock $\operatorname{ELP}_{\mathrm{BC}} \mathrm{S}$ that vary in the hydrophobic-hydrophilic segment ratio (SR) to (1) demonstrate thermal self-assembly as a trigger for receptor-mediated binding activity and (2) identify the optimal nanoscale architecture for multivalent interaction of the micelles with the $\alpha_{v} \beta_{3}$ integrin.

\section{RESULTS}

We chose parent $E_{\mathrm{E}} \mathrm{P}_{\mathrm{BC}} \mathrm{S}$ that were available from previous studies ${ }^{10,21}$ to examine the size and nanoscale architecture of their micelles on DAM. Each ELP $\mathrm{BC}_{\mathrm{BC}}$ (defined hereafter as ELP-Y/Z) comprises a hydrophilic block of $Y$ VPGXG repeats (where $X=V / A / G$ in a 1:7:8 ratio) and a hydrophobic block of $Z$ VPGVG repeats. We chose three separate $\operatorname{ELP}_{\mathrm{BC}} \mathrm{S}$ for this study, ELP-96/60, ELP-64/60, and ELP-64/90, that span a SR range of 0.66 to 1.5 (Figure $1 \mathrm{~B}$ ). We have previously shown that each of these $E \mathrm{P}_{\mathrm{BC}} \mathrm{S}$ self-assembles into micelles in response to an increase in solution temperature, ${ }^{10}$ and this range of SRs allowed us to study the effect of nanoscale architecture on multivalent interaction between the ligand and receptor. Two variants of homopolymeric ELP that comprise 150 VPGXG (where $X=V / A / G$ in a 5:3:2 ratio, 

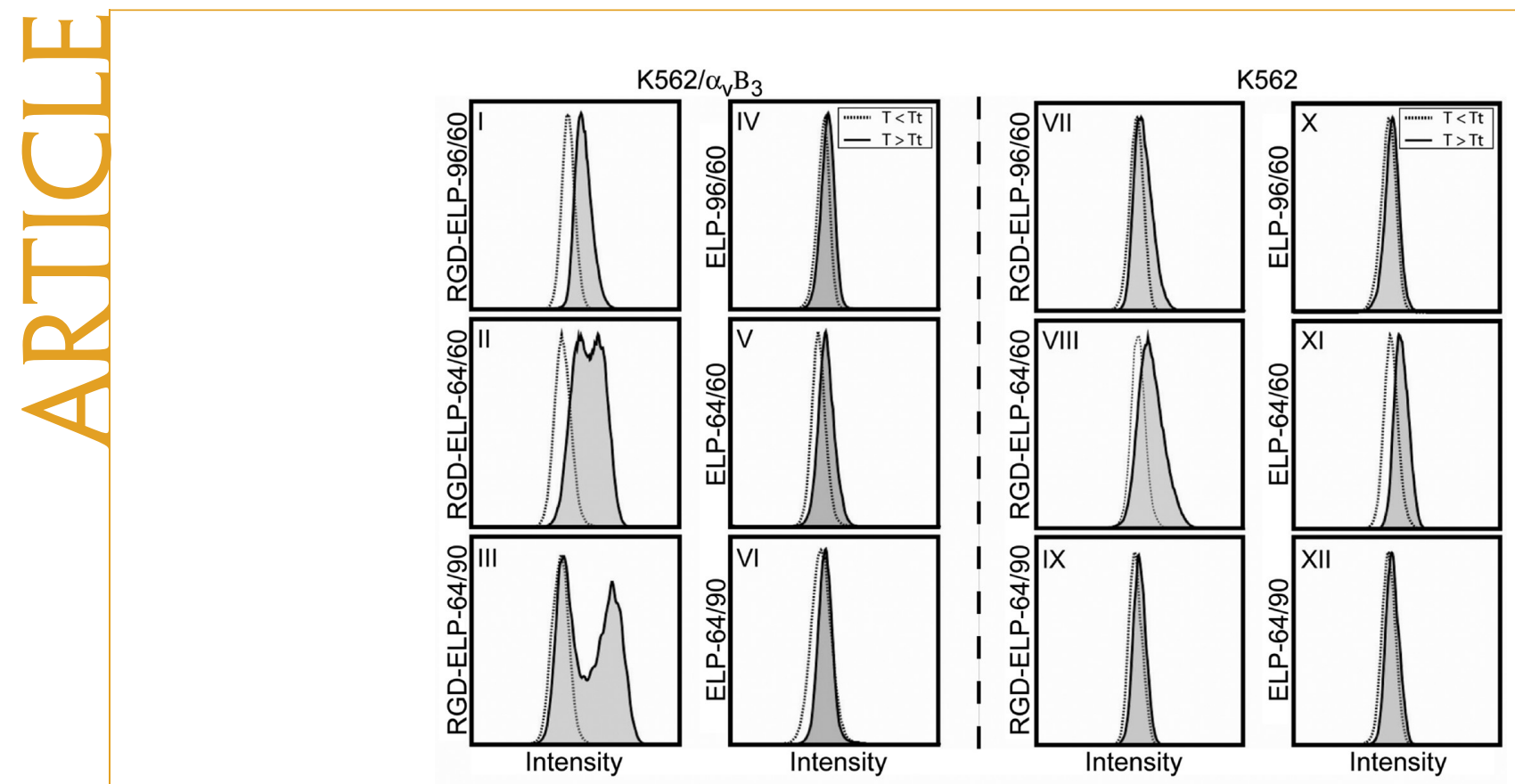

Figure 3. Flow cytometry analysis of $\mathrm{K} 562$ and $\mathrm{K} 562 / \alpha_{\mathrm{v}} \boldsymbol{\beta}_{3}$ cells following incubation with $10 \mu \mathrm{M}$ RGD-ELP-64/90 or ELP$64 / 90$ below (dashed line) and above (solid line) the CMT. There was no significant difference in the histograms of any of the cell populations incubated below the CMT. Neither cell line showed enhanced binding/uptake of the ELP $\mathrm{BC}_{\mathrm{BC}} \mathrm{S}$ above their CMT, as seen by their similar flow cytometry histograms (panels IV-VI and X-XII), and the RGD-ELP $\mathrm{BC}_{\mathrm{BC}}$ did not show appreciable interaction with $\mathrm{K} 562$ cells above the CMT (panels VII-IX). There was a slight increase in binding/uptake of RGD-ELP$96 / 60$ above its CMT by $\mathrm{K} 562 / \alpha_{\mathrm{v}} \beta_{3}$ cells, but there was a dramatic increase in the fraction of $\mathrm{K} 562 / \alpha_{\mathrm{v}} \boldsymbol{\beta}_{3}$ cells that take up RGD-ELP-64/60 and RGD-ELP-64/90 above their CMT (panels I-III). This of this second peak increasingly shifted right with increasing SR of each ELP $P_{B C}$ indicating greater levels of interaction per cell.

ELP-150) were also synthesized, one of which has a terminal RGD peptide and negative control lacking the RGD peptide, serving as controls to examine the effect of nanoscale presentation.

$E P_{B C} S$ were modified at the gene level to attach a GRGDS ligand at their N-terminus and a unique cysteine residue on their $\mathrm{C}$-terminus. Each modified ELP $\mathrm{P}_{\mathrm{BC}}$ was expressed from a plasmid-borne synthetic gene in E. coli which encoded three contiguous segments: an $\mathrm{N}$-terminal GRGDS peptide ligand, the $\mathrm{ELP}_{\mathrm{BC}}$, and a short WPC peptide that provides a unique cysteine residue for conjugation of a fluorophore or drug at the C-terminus (Figure 1B). ELP $\mathrm{BC}_{\mathrm{BC}}$ that contained the C-terminal WPC sequence but not the $\mathrm{N}$-terminal GRGDS ligand served as a negative control for the effect of ligand presentation.

Each ELP was recombinantly synthesized by inserting each parent ELP $_{\mathrm{BC}}$ gene into a modified $\mathrm{pET} 25 \mathrm{~b}$ expression vector (Novagen, Madison, WI) with subsequent overexpression of the $E_{\mathrm{E}} \mathrm{P}_{\mathrm{BC}}$ genes in E. coli. Agarose gel electrophoresis and DNA sequencing demonstrated successful cloning of RGD-modified ELP $P_{B C}$ and ELP genes lacking the RGD peptide into expression vectors (data not shown). All constructs were expressed from their plasmid-borne genes in E. coli at high yields ( $>50 \mathrm{mg} / \mathrm{L}$ in shaker flask culture) and were purified by inverse transition cycling (ITC). ${ }^{12}$ SDS-PAGE showed that the ELPs were monodisperse and were purified to homogeneity by ITC. The purified ELPs were then conjugated to AlexaFluor488 (Invitrogen, Carls- bad, CA) by reaction between the maleimide moiety of the fluorophore and the terminal cysteine of the $\operatorname{ELP}_{\mathrm{BC}} \mathrm{S}$ and ELP-150 with a yield of $60-70 \%$. Labeled constructs were used for all subsequent experiments.

We characterized the thermally triggered selfassembly of the $\operatorname{ELP}_{\mathrm{BC}} \mathrm{S}$ by dynamic light scattering (DLS). We monitored the hydrodynamic radius $\left(R_{h}\right)$ and coordination number $(Z)$ of a $10 \mu \mathrm{M}$ ELP solution as a function of temperature. Both RGD-ELP $P_{B C}$ and the parent $E P_{B C}$ exhibited two phases as the solution temperature was raised from 20 to $42{ }^{\circ} \mathrm{C}$ : (1) a soluble unimer phase with a $R_{\mathrm{h}}=5-8 \mathrm{~nm}$ and (2) a nanoparticle with a $R_{\mathrm{h}}$ of $\sim 30 \mathrm{~nm}$ at higher temperatures (Figure 2). The coordination number ( $Z$ ), defined as the number of unimers comprising one micelle, was determined for each construct and allowed estimation of the ligand density of RGD moieties in the corona, which was similar for all RGD-ELP $P_{B C}$ constructs of different SRs (Figure 2). This ligand density is within the range of densities required for multivalent RGD $-\alpha_{v} \beta_{3}$ interaction ${ }^{22}$ and supports the use of these constructs for multivalent targeting of the $\alpha_{v} \beta_{3}$ integrin.

After verifying thermally triggered self-assembly, we examined the feasibility of DAM using the $\operatorname{ELP}_{\mathrm{BC}} \mathrm{S}$ as the thermosensitive carrier, linear RGD peptide as the ligand, AlexaFluor488 as the surrogate for an imaging agent or drug, and mild hyperthermia as the thermal switch. For DAM to be successful using RGD-ELP $P_{B C} S$, four requirements must be met. First, $R G D-E L P_{B C}$ must exist in an "off" state below its CMT; it must not 
interact with cellular receptors in its hydrophilic, monovalent state. This would ensure that RGD-ELP $\mathrm{BC}_{\mathrm{BC}}$ does not interact with cell receptors outside the target area. Second, self-assembly of ELP $\mathrm{BC}_{\mathrm{BC}}$ into micelles must not promote cellular interaction independent of ligand presentation. This is an important consideration as it would allow the $E P_{B C}$ to act as a scaffold for multivalent ligand presentation without directly enhancing nonspecific binding or uptake by cells. Third, RGD-ELP $P_{B C}$ must exist in an "on" state above the CMT; RGD-ELP $P_{B C}$ micelles should lead to enhanced interaction with receptorpositive cells compared to receptor-negative cells. Meeting this last requirement would prove the ability for controlled multivalency to act as a trigger for receptor-specific cell interaction. Fourth, the $\mathrm{RGD}-\mathrm{ELP}_{\mathrm{BC}}$ micelle must provide the optimal nanoscale architecture to allow multivalent interactions with cell surface receptors.

To test the four requirements for DAM, we measured cellular binding and uptake of the ligandfunctionalized RGD-ELP $P_{B C}$ and corresponding ligandnegative $E L P_{B C}$ controls by wild-type $\mathrm{K} 562$ human leukemia cells (receptor-negative control) and K562 cells transformed with the $\alpha_{\mathrm{v}} \beta_{3}$ receptor $\left(\mathrm{K} 562 / \alpha_{\mathrm{v}} \beta_{3}\right){ }^{23}$ Briefly, $10 \mu \mathrm{M}$ of either AlexaFluor488-labeled $\mathrm{RGD}-\mathrm{ELP}_{\mathrm{BC}}$ or $\mathrm{ELP}_{\mathrm{BC}}$ were incubated with $\mathrm{K} 562$ or K562/ $\alpha_{\mathrm{v}} \beta_{3}$ for $1 \mathrm{~h}$ at $T<\mathrm{CMT}\left(T=23^{\circ} \mathrm{C}\right)$ or $T>\mathrm{CMT}(T=$ $40^{\circ} \mathrm{C}$ ). These temperatures were chosen to ensure consistent temperatures for monovalent and multivalent presentation of all constructs. The cells were then analyzed for fluorescence intensity using flow cytometry (Figure 3). Each panel corresponds to a unique pair of $E_{B C}$ and cell line, and the data in each panel are normalized to the flow cytometry distribution for that ELP $P_{B C}$ below its CMT (dashed distributions). The relevant data are the increase in fluorescence for the $E_{\mathrm{LPC}}$ above the CMT (distribution in gray) relative to its cell uptake/ binding below its CMT. Cell fluorescence, a measure of $E P_{B C}$ binding and uptake, was then quantified using flow cytometry histograms to determine the potential of $E P_{B C}$ to exhibit DAM. These results were independently verified with confocal microscopy (Figure 4).

We first evaluated the effects of monovalent ligand presentation on specific cell interaction by incubating $\mathrm{RGD}-\mathrm{ELP}_{\mathrm{BC}}$ or $\mathrm{ELP}_{\mathrm{BC}}$ with both cell types below the CMT and analysis by flow cytometry. The distribution of each cell population incubated below the CMT is designated by dashed lines in each panel (Figure 3). As seen in the fluorescence histograms, there was little difference between the fluorescence intensity of any of the cell populations incubated with either ELP $\mathrm{P}_{\mathrm{BC}}$ below the CMT. Confocal fluorescence images supported these findings as there was no visible fluorescence in cells in-

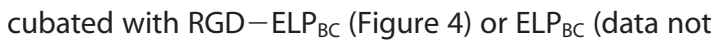
shown) below the CMT. These results suggest that the $\mathrm{K} 562$ and $\mathrm{K} 562 / \alpha_{\mathrm{v}} \beta_{3}$ cell lines exhibit low levels of nonspecific binding and uptake of the ELPs under these ex-

www.acsnano.org perimental conditions in contrast to other cell lines that show some, albeit low, levels of interaction with ELP. ${ }^{21,24}$ These data also indicate that monovalent presentation of RGD by ELP $P_{B C}$ is not sufficient to promote cell interaction beyond that of the parent $\operatorname{ELP}_{B C}$, satisfying one criterion for DAM.

We next evaluated the effect of self-assembly on nonspecific cellular binding and uptake (i.e., in the absence of the RGD ligand). We incubated ligand-negative control polymers below and above their CMT with $\mathrm{K} 562$ and $\mathrm{K} 562 / \alpha_{\mathrm{v}} \beta_{3}$ cells, respectively, and analyzed specific cell interaction of the ELPs by flow cytometry (Figure 3). We quantified these differences by first obtaining a histogram corresponding to unheated cells and defining the region two standard deviations (SD) above the mean fluorescence as a significant increase in fluorescence intensity (AlexaFluor488 ${ }^{+}$). This region was identified in each histogram of heated cells incubated with the same concentration of ELP $\mathrm{BC}_{\mathrm{BC}}$. The fraction of heated cells within this region was used as one metric to quantify the effects of self-assembly on cellular interaction (Figure $5 \mathrm{~A}$ ).

These data reveal that the effect of heat on cell binding and uptake of ELP $_{\mathrm{BC}} \mathrm{S}$ was similar in either cell line. The flow cytometry data show that the distribution of fluorescence intensities did not significantly change at $T>$ CMT relative to $T<$ CMT in either cell line. Quantitatively, only $<10 \%$ of the heated cells showed significantly higher fluorescence intensity than the unheated control. These results clearly show that the cells did not interact more with control ELP at $T>$ CMT compared to the same ELP at $T<C M T$ and thus indicate that the effect of temperature-triggered self-assembly on cell interaction was minimal for both cell lines in the absence of the RGD ligand (Figure 5A). Confocal images of $E P_{B C} S$ in both cell types visually confirmed the absence of significant fluorescence both below and above the CMT (Figure 4), corroborating the flow cytometry data. Clearly, micelle formation by itself does not significantly promote cellular binding and uptake, demonstrating that $\mathrm{ELP}_{\mathrm{BC}}$ can act as an inert scaffold for multivalent presentation of targeting ligands.

Next, we evaluated the effect of presentation of ligand to determine if multivalent presentation of the RGD ligand promotes receptor-mediated binding. Prior to these experiments, we quantified integrin expression levels in $\mathrm{K} 562$ and $\mathrm{K} 562 / \alpha_{\mathrm{v}} \beta_{3}$ cell lines by antibody (Ab) staining with a fluorescently labeled, $\alpha_{v} \beta_{3}$-specific $\mathrm{Ab}$ (LM609) and flow cytometry analysis (Supporting Information Figure 1). This experiment revealed a bimodal distribution of integrin expression, with only $\sim 60 \%$ of $\mathrm{K} 562 / \alpha_{\mathrm{v}} \beta_{3}{ }^{+}$cells expressing the receptor. Interestingly, there was also low $(\sim 15 \%) \alpha_{\mathrm{v}} \beta_{3}$ expression on the receptor-negative $\mathrm{K} 562$ cells (Figure $5 \mathrm{~A}$ ). These findings suggested that if the RGD-ELP $P_{B C}$ specifically interacts with the $\alpha_{v} \beta_{3}$ integrin, then resulting histograms of the RGD-ELP $\mathrm{BC}_{\mathrm{B}} \mathrm{S}$ by receptor-positive $\mathrm{K} 562 / \alpha_{\mathrm{v}} \beta_{3}$ cells 
(A)
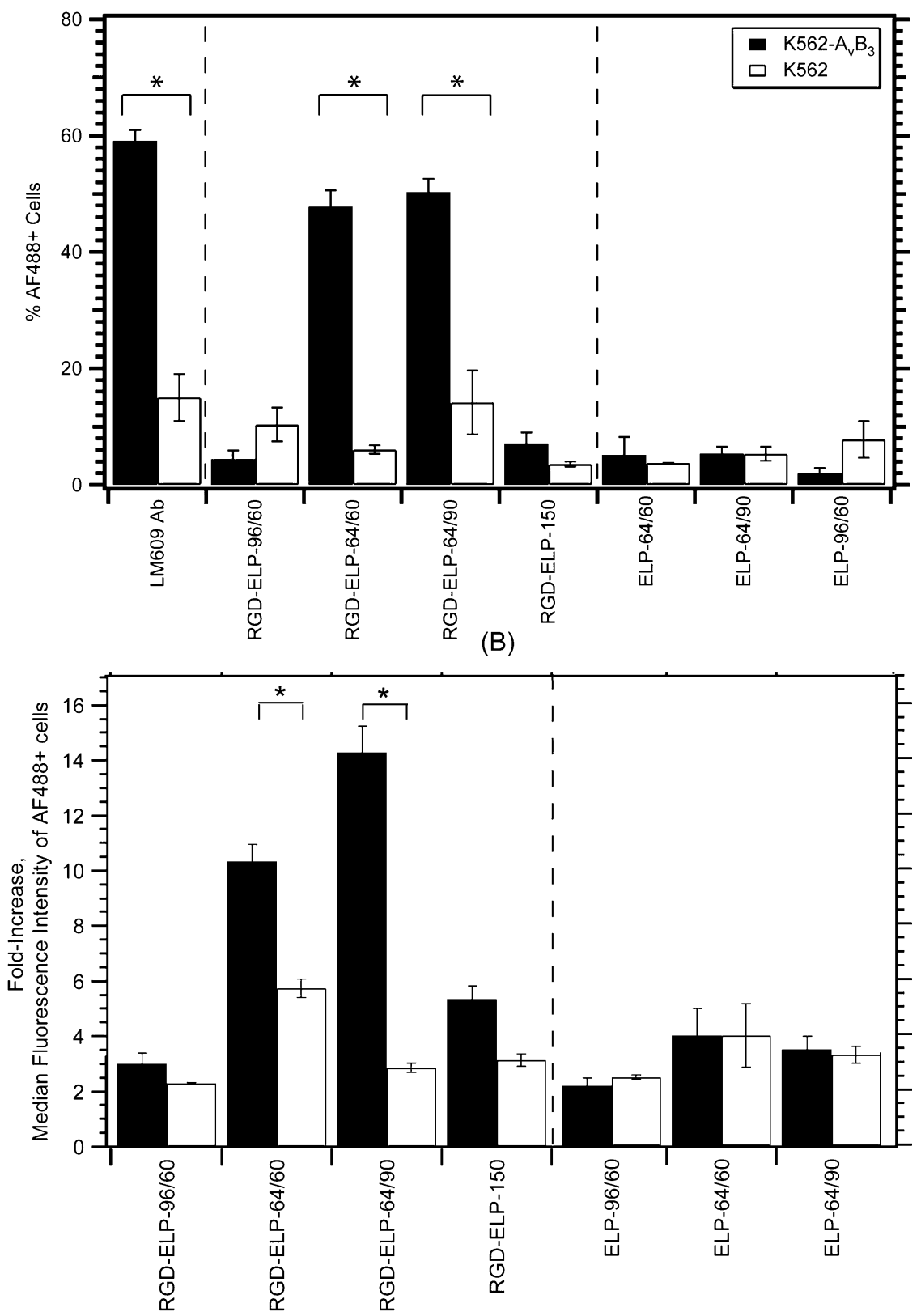

Figure 4. Analysis of segment ratio (SR) on cellular binding/uptake. (A) Approximately $60 \%$ of $K 562 / \alpha_{v} \beta_{3}$ were positive for the $\alpha_{\mathrm{v}} \boldsymbol{\beta}_{3}$ receptor, as seen by the binding of the LM609 antibody that is specific for the $\boldsymbol{\alpha}_{\mathrm{v}} \boldsymbol{\beta}_{3}$ integrin. The percentage of AlexaFluor488 ${ }^{+}$cells increased to $50-60 \%$ relative to unheated controls when RGD-ELP-64/90 and RGD-ELP-64/60 were incubated with $\mathrm{K} 562 / \boldsymbol{\alpha}_{\mathrm{v}} \boldsymbol{\beta}_{3}$, similar to the $\%$ of $\boldsymbol{\alpha}_{\mathrm{v}} \boldsymbol{\beta}_{3}$ cells. There was no significant increase in \% AlexaFluor488 ${ }^{+}$cells with heating for any other combination of construct and cells. (B) Fold increase in median fluorescence of AlexaFluor488 ${ }^{+}$cells was measured for each cell/construct combination. There was a small increase in fluorescence of both cell lines incubated with the parent ELP $\mathrm{BC}_{\mathrm{BC}} \mathrm{S}$ and of K562 cells that were incubated with RGD-ELPBC. The median fluorescence of $\boldsymbol{\alpha}_{\mathrm{v}} \boldsymbol{\beta}_{3}$ increased with RGD-ELP-64/60 and RGD-ELP-64/90 above their CMT, while there was a slight increase in binding of RGD-ELP-150 and RGD-ELP-96/60 by $\boldsymbol{\alpha}_{\mathrm{v}} \boldsymbol{\beta}_{3}$. The median fluorescence also increased with increasing SR of the RGD-ELP ${ }_{B C}$ s. Groups that exhibited a significant difference are denoted by $*(p<.05)$.

should mirror those observed for the LM609 antibody. Hence, $\mathrm{K} 562 / \alpha_{v} \beta_{3}$ cells incubated with RGD-ELPBC at $T$ $>$ CMT should also show increased fluorescence with a bimodal distribution. In contrast, reflective of the low level of receptor expression by $\mathrm{K} 562$ cells, RGD-ELP $\mathrm{BC}_{\mathrm{B}}$ interaction with the receptor-negative K562 cell line should only show a slight $(<15 \%)$ increase at $T>$ CMT as compared to $T<$ CMT.
RGD - ELP $P_{B C} S$ were next incubated with $\mathrm{K} 562$ and K562/ $\alpha_{v} \beta_{3}$ cells at $T<$ CMT and $T>$ CMT for $1 \mathrm{~h}$. Each cell population was then monitored by flow cytometry to determine the effects of self-assembly and ligand-receptor interaction on cell binding/uptake. The resulting data show two interesting features. First, all $R G D-E L P_{B C S}$ led to a small increase in fluorescence intensity above the CMT as compared to the same ELP 
(A)
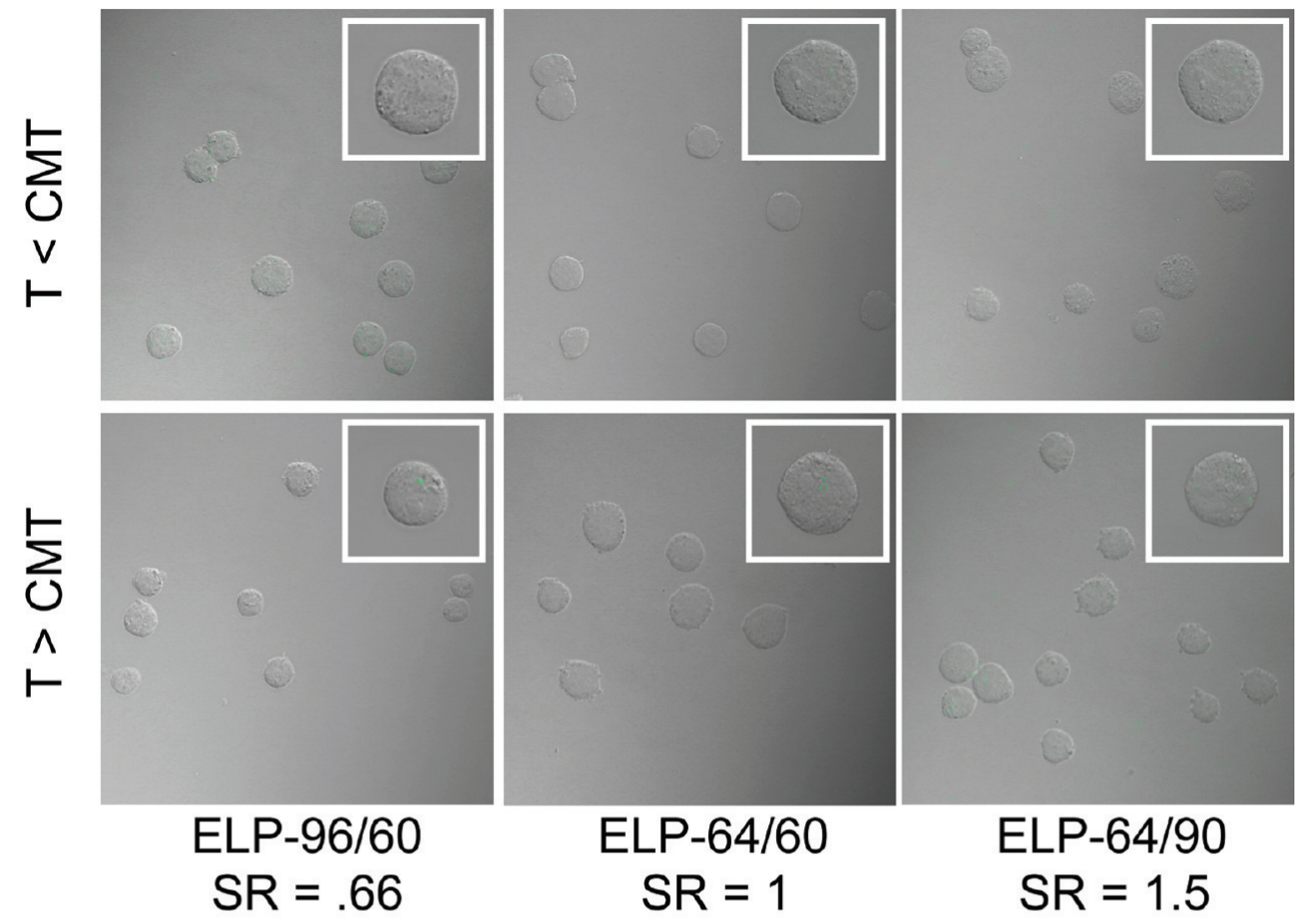

ELP-64/60

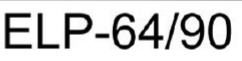

$\mathrm{SR}=1$

$\mathrm{SR}=1.5$

(B)
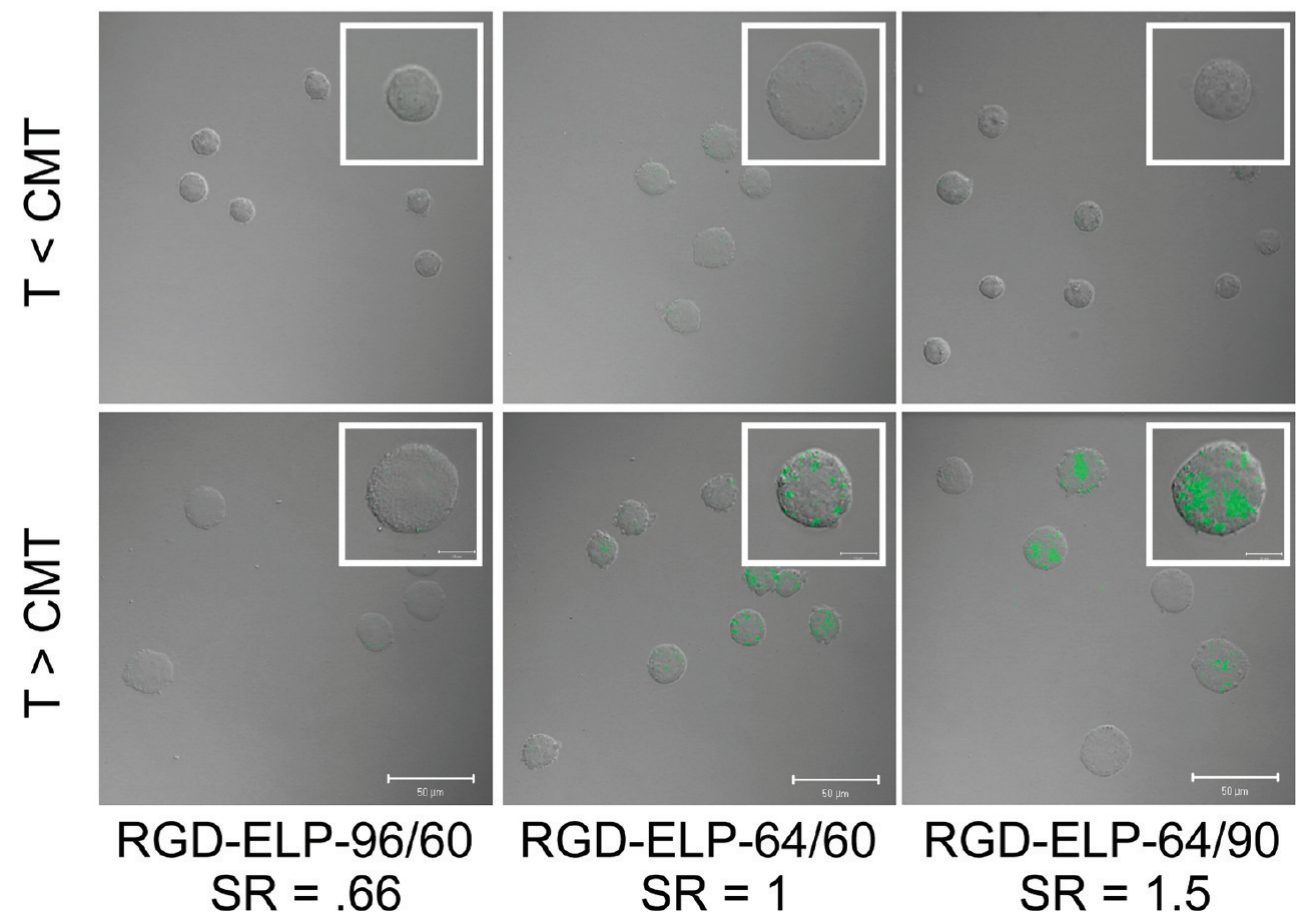

RGD-ELP-64/60 $\mathrm{SR}=1$

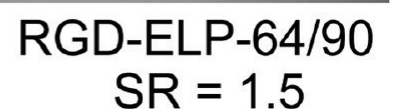

Figure 5. Confocal fluorescence images of $K 562 / \alpha_{\mathrm{v}} \beta_{3}$ cells following incubation with $10 \mu \mathrm{M}$ of various ELP $\mathrm{B}_{\mathrm{BC}} \mathrm{S}$ (green). (A) There was no visible binding of any of the ELP $P_{B C}$ by $K 562 / \alpha_{v} \beta_{3}$ cells when they were incubated below and above the CMT with the ligand-negative $\mathrm{ELP}_{\mathrm{BC}}$ controls, demonstrating that micelle formation alone does not promote nonspecific interaction. (B) There was also minimal visible binding/uptake of all three RGD-ELP ${ }_{B C}$ below or above the CMT when incubated with $\mathrm{K} 562$ cells, showing that overexpression of the receptor is necessary for enhanced interaction. There was no visible binding/uptake of RGD-ELP-64/60 or RGD-ELP-64/90 below the CMT by K562/ $\boldsymbol{\alpha}_{v} \boldsymbol{\beta}_{3}$ cells, but there was significant binding/uptake of RGD-ELP-64/60 or RGD-ELP-64/90 above their CMT. A binary population of highly fluorescent and nonfluorescent cells in the field of view was observed by fluorescence microscopy, corresponding to the bimodal distribution seen in the flow cytometry histogram in panels II and III in Figure 3 . Size bar $=50$ $\mu \mathrm{m}$. 
below the CMT in the receptor-negative K562 cell line (panels IV-VI, Figure 3). Quantification of this effect showed that $5-15 \%$ of cells showed significantly higher fluorescence intensity following heating (Figure $4 \mathrm{~A})$. This result is consistent with the finding that $<20 \%$ of the cells in the K562 cell line express the $\alpha_{v} \beta_{3}$ integrin and indicates that there is slightly enhanced interaction of RGD-ELP $P_{B C}$ in its micellar state $(T>C M T)$ compared to the monovalent state $(T<\mathrm{CMT})$ by $\mathrm{K} 562$ $\left(\alpha_{\mathrm{v}} \beta_{3}{ }^{-}\right)$cells.

Second, the histograms of the receptor-positive $\mathrm{K} 562 / \alpha_{\mathrm{v}} \beta_{3}$ cells incubated with RGD-ELP $\mathrm{BC} \mathrm{S}$ (panels I-III, Figure 3) were dramatically different based on the ELPBC SR. The fluorescence histogram of micellar RGD-ELP- $96 / 60$ by $K 562 / \alpha_{v} \beta_{3}$ cells was similar to wildtype K562 cells, illustrating that multivalent ligand presentation did not have an effect on cell receptor presentation for this $\mathrm{ELP}_{\mathrm{BC}}$. In contrast, the RGD-ELP-64/60 and RGD-ELP-64/90 micelles ( $T>C M T$ ) both exhibited a bimodal distribution of fluorescence, with $\sim 50 \%$ of the $\mathrm{K} 562 / \alpha_{\mathrm{v}} \beta_{3}$ cells exhibiting a significant increase in fluorescence intensity relative to the same ELPs in unimer form ( $T<\mathrm{CMT}$ ) (Figure 3). Confocal fluorescence microscopy of $\mathrm{K} 562 / \alpha_{\mathrm{v}} \beta_{3}$ cells incubated with RGD-ELP $P_{B C} S$ confirmed these results (Figure $5 B$ ). While K562/ $\alpha_{\mathrm{v}} \beta_{3}$ cells incubated with RGD-ELP-96/60 did not show significant interaction with ELP, cells incubated with RGD-ELP-64/60 or RGD-ELP-64/90 above their CMT showed a binary distribution with an approximately equal fraction of cells exhibiting high fluorescence and another population that exhibited virtually no fluorescence (Figure 5B). These results clearly suggest that a threshold of SR is required, above which there is significant receptor-mediated interaction of the $\mathrm{RGD}-\mathrm{ELP}_{\mathrm{BC}}$ with $\mathrm{K} 562 / \alpha_{\mathrm{v}} \beta_{3}$ cells.

In addition, there was a noticeable right shift of the peak within the AlexaFluor488 ${ }^{+}$region of the flow cytometry histograms, representing a significant difference in the per-cell fluorescence intensity of cells targeted by RGD-ELP-64/90 and RGD-ELP-64/60 compared to RGD-ELP-96/60 (Figure 3). Quantitative analysis of this shift showed a significant increase in the median fluorescence intensity of this peak with increasing SR of the ELP $\mathrm{BC}_{\mathrm{BC}}$ (Figure 4B). The normalized intensity of this peak following incubation with RGD-ELP64/90 was 5 -fold greater at $T>$ CMT than at $T<$ CMT, while a 2-fold increase in the intensity of this peak was observed for RGD - ELP-64/60 at $T>$ CMT relative to $T<$ CMT. These results were visually confirmed by confocal fluorescence microscopy (Figure 5B). K562/ $\alpha_{\mathrm{v}} \beta_{3}$ cells incubated with RGD-ELP-64/90 at $T>$ CMT showed greater fluorescence than RGD-ELP-64/60, which, in turn, showed greater fluorescence than RGD-ELP$96 / 60$. These data clearly suggest that an increase in hydrophobic content of the RGD-ELP $P_{B C}$, as defined by their SR, enhances multivalent ligand-receptor interaction following micelle formation.
We further evaluated the importance of nanoscale architecture in controlling the receptor-mediated interaction of the RGD-terminated ELP ${ }_{B C} \mathrm{~S}$. To do so, we expressed an RGD-ELP-150, a homopolymer that exhibits inverse phase transition behavior. RGD-ELP-150 has approximately the same MW as the $\operatorname{ELP}_{\mathrm{BC}} \mathrm{S}$ used in this study, but this ELP exhibits completely different temperature-dependent behavior at the same solution concentration as the ELP $P_{B C}$. RGD-ELP-150 is soluble at $T<\mathrm{T}_{\mathrm{t}}$ and forms micron-scale aggregates at $T>T_{\mathrm{t}}$, as confirmed by DLS (Supporting Information Figure 2). Because RGD-ELP-150 forms micron-scale aggregates rather than nanoscale micelles in the temperature range of interest $\left(20-42^{\circ} \mathrm{C}\right)$, it is useful for determining the importance of ligand presentation on a nanoscale scaffold as opposed to a polydisperse aggregate. We incubated RGD-ELP-150 with both K562 and K562/ $\alpha_{v} \beta_{3}$ cells at $T>T_{\mathrm{t}}$. The fraction of AlexaFluor488 ${ }^{+}$ $\mathrm{K} 562$ and $\mathrm{K} 562 / \alpha_{\mathrm{v}} \beta_{3}$ cells following incubation at $T>$ $T_{\mathrm{t}}$ was significantly smaller than $\mathrm{K} 562 / \alpha_{\mathrm{v}} \beta_{3}$ cells incubated with RGD-ELP-64/90 or RGD-ELP-64/60 (Figure 5) above its CMT. These results indicate that multivalent presentation by a ordered nanoscale scaffold led to greater cell binding/uptake as compared to presentation of the same ligand by a large polydisperse aggregate, thus highlighting the importance of the nanoscale architecture of ligand presentation for multivalent targeting.

\section{DISCUSSION}

The results presented herein demonstrate the feasibility of DAM by temperature-triggered self-assembly of a ligand-functionalized, genetically encoded diblock $E P_{B C}$. Our results show that multivalent presentation of the RGD peptide ligand by self-assembled RGD-ELP ${ }_{B C}$ nanoparticles promotes significant binding of the polymer only to cells that overexpress the $\alpha_{v} \beta_{3}$ integrin. In contrast, both receptor-positive and receptor-negative cell lines show low binding of RGD-ELP $P_{B C}$ in its lowaffinity, monovalent state. Both cell lines also show low binding of ligand-negative $\operatorname{ELP}_{\mathrm{BC}}$ nanoparticles following their temperature-triggered self-assembly.

Given that the cellular binding/uptake is only significantly greater for multivalent RGD-ELP $\mathrm{BC}_{\mathrm{BC}}$ constructs in receptor-positive cells as compared to all other negative controls, the cause for increased cellular interaction by RGD-ELP $P_{B C}$ is likely caused by higher avidity of the multivalent RGD micelle compared to the lower affinity of the monovalent RGD-ELP $P_{B C}$. Our data further indicate that this multivalent presentation of the RGD ligand requires an ordered scaffold such as the $\mathrm{ELP}_{\mathrm{BC}}$ micelle, as polydisperse, micrometer-sized aggregates of RGD-ELP-150 did not show enhanced interaction above their $T_{\mathrm{t}}$. These observations also suggest that that large fluorescence aggregates observed in the images of $\mathrm{K} 562 / \alpha_{\mathrm{v}} \beta_{3}$ are a result of integrin clusters in close proximity rather than aggregated ELP. ${ }^{25}$ 
An interesting finding of this study was that $\mathrm{RGD}-\mathrm{ELP}_{\mathrm{BC}} \mathrm{S}$ with higher hydrophobic content (and hence a larger SR) are more avidly interact with cells that overexpress the $\alpha_{\mathrm{v}} \beta_{3}$ receptor. This finding is notable as it suggests that subtle differences in the molecular architecture of a nanoscale ligand scaffold can have a large effect on receptor binding. To the best of our knowledge, the effect of this level of architectural control on ligand presentation has not been uncovered in previous studies of receptor-mediated binding by self-assembled polymeric micelles.

Although the origins of this behavior are not clear at this time, we believe that a likely explanation of binding dependence on SR is ligand-receptor accessibility. Although future study is needed to establish a definite mechanism, we believe that RGD-ELP $P_{B C}$ micelles with larger hydrophobic cores may have subtle differences in the mobility of the terminal ligand that enable more effective presentation of multiple ligands to membrane-bound $\alpha_{\mathrm{v}} \beta_{3}$ receptors. This hypothesis is consistent with our previous work showing different patterns of micelle formation that correlated with the $\mathrm{SR}$ of the $\mathrm{ELP}_{\mathrm{BC}}{ }^{10}$ We observed a decrease in the apparent stability of the nanoparticles with increased SR of the $E_{\mathrm{EP}} \mathrm{BC}$ as evidenced by the smaller temperature range over which monodisperse micelles are the predominant phase. ${ }^{10}$ The decrease in micelle stability, we suggest, may correlate with greater mobility within the corona of the micelle and thus greater simultaneous accessibility to different $\alpha_{v} \beta_{3}$ integrins. Additionally, multivalent interaction involving the $\alpha_{\mathrm{v}} \beta_{3}$ integrin requires receptor clustering following integrin activation. ${ }^{20}$ ELP$64 / 90$, which showed the greatest cellular interaction of the three $\mathrm{ELP}_{\mathrm{BC}} \mathrm{S}$ studied herein, shows a steady increase in size with temperature. This may also facilitate cluster formation due to the greater probability of covering multiple integrins prior to clustering. While additional studies are needed to fully understand the reasons behind these differences in binding, there appears to be a clear effect of micelle architecture on its multivalent interaction with a specific cell surface receptor.

Finally, these biopolymers have other ancillary attributes that make them attractive for the targeted de- livery of drugs and imaging agents. They can be readily overexpressed from a synthetic gene with a low-affinity peptide ligand appended at their hydrophilic terminus and unique reactive sites for conjugation of drugs or imaging agents at the hydrophobic end of the polymer, ensuring convenient synthesis. They are readily purified with sufficient yield and high purity by means of their phase transition behavior. The $\operatorname{ELP}_{\mathrm{BC}} \mathrm{S}$ are also monodisperse and exhibit a precisely defined nanoscale architecture following self-assembly. Finally, the thermally triggered micelle self-assembly of these $\mathrm{ELP}_{\mathrm{BC}} \mathrm{S}$ is retained in serum (Supporting Information Figure 4), suggesting that these polymers will retain their targeting properties following systemic in vivo administration. To the best of our knowledge, this is the first example of a rationally designed polymer that exhibits dynamic modulation of receptor binding affinity in response to an external trigger.

Although these results are promising for dynamic multivalent targeting, they represent only the first step toward translation of these findings into a nanoscale carrier that will have clinical utility to target specific tissues and organs. The first and outstanding challenge is to re-engineer the RGD-ELP-64/90, which exhibited the largest magnitude of DAM, to exhibit a CMT between 39 and $43^{\circ} \mathrm{C}$ under physiological conditions, the current approved temperature range for mild hyperthermia. ${ }^{26}$ RGD-ELP-64/90 shows self-assembly into nanoparticles in serum with a CMT of $33^{\circ} \mathrm{C}$ (Supporting Information Figure 3), which is $7{ }^{\circ} \mathrm{C}$ lower than the target CMT of $40^{\circ} \mathrm{C}$. We believe, based on our previous experience in designing ELPs, that moving the CMT of this $E P_{B C}$ into the desired range for mild clinical hyperthermia should be possible by a subtle alteration of the guest residue composition of the hydrophobic block without compromising its self-assembly or ligand presentation. The second challenge is to select a drug that does not perturb the self-assembly process. In this regard, we believe that a rational strategy is to match the hydrophobicity of the drug with that of the hydrophobic core. Future studies will focus on addressing these challenges to move DAM into a preclinical animal model.

\section{METHODS}

Nomenclature. ELPs are described by the nomenclature ELP $\mathrm{V}_{x}$ $\left.A_{y} G_{z}\right]_{m}$, where $m$ refers to the number of pentapeptide repeats and $x, y$, and $z$ refer to the relative fraction of valine, alanine, and glycine in the guest residue position along the length of the protein, respectively. The number in the shorthand ELP description refers to the number of pentapeptide repeats in the particular segment. All block copolymers used in this study have the composition ELP $\left[V_{1} A_{8} G_{7}\right] / E L P\left[V_{5}\right]$, and the homopolymer has the composition ELP $\left[V_{5} A_{2} G_{3}\right]$. For example, the diblock copolymer ELP-64/90 consists of two blocks; the first is composed of 64 pentapeptides and the second of 90 pentapeptides. In contrast, the ELP-150 consists of 150 pentapeptides.
ELP Cloning and Expression. The ELP $\left[\mathrm{V}_{1} \mathrm{~A}_{8} \mathrm{G}_{7}\right] /$ ELP $\left[\mathrm{V}_{5}\right]$ block copolymer gene and the ELP $\left[V_{5} A_{2} G_{3}\right]$ gene were synthesized using the recursive directional ligation method described previously. ${ }^{7}$ Unmodified pET-25b plasmid was digested with EcoRI and Ndel (New England Biolabs, Ipswich, MA) and purified using a gel extraction kit (Qiagen, Valencia, CA). Synthetic oligonucleotides encoding the sense and antisense strands of the $\mathrm{N}$-terminal leader and $\mathrm{C}$-terminal trailer peptide sequences in the ELPs (IDT, Coralville, IA) were annealed to form a cassette with EcoRI- and Ndel-compatible ends. These cassettes were ligated into EcoRI/Ndel-digested pET-25b and transformed into Top10 competent cells (Invitrogen, La Jolla, CA) to create the modified pET-25bAS2 and pET-25bSV2 expression vectors (Supporting Information Figure 4). Following confirmation by 
restriction digestion, the $\mathrm{pET}-25 \mathrm{bAS} 2$ and $\mathrm{pET}-25 \mathrm{bSV} 2$ vectors were digested with Sfil (New England Biolabs, Ipswich, MA) and purified by gel purification, as above. The gene corresponding to ELP-64/90 was ligated into both modified pET-25b vectors and transformed into BLR competent cells (Novagen, Madison, WI). The insertion of the ELP-64/90 and ELP-150 genes into each vector was confirmed by gel electrophoresis of plasmids digested with Xbal and HindIII (New England Biolabs, Ipswich, MA) followed by DNA sequencing.

ELP Purification. All ELPs used in this study were expressed by a hyperexpression protocol as follows: BLR E. coli containing the ELP plasmid were grown overnight in a shaker incubator in 50 $\mathrm{mL}$ of TB dry media (Mo Bio Laboratories, Inc., Carlsbad, CA) and $1 \mathrm{mg} / \mathrm{mL}$ of ampicillin at $37^{\circ} \mathrm{C}$ and $270 \mathrm{rpm}$. The resulting cultures were centrifuged to collect the cells, and the cell pellet was resuspended and grown overnight in $1 \mathrm{~L}$ of TB dry media with $1 \mathrm{mg} / \mathrm{mL}$ of ampicillin at $37{ }^{\circ} \mathrm{C}$ and $270 \mathrm{rpm}$. The cells were harvested for the culture and lysed, and the ELP was then purified using the inverse transition cycling (ITC) purification method as previously described. ${ }^{12}$ Each ELP was purified from the soluble fraction of cell lysate by five rounds of ITC, then resuspended in PBS, and stored at $-20^{\circ} \mathrm{C}$ until further use.

Fluorophore Conjugation. One milliliter of $200 \mu \mathrm{M}$ ELP (all ELPs in this study) was pelleted by centrifugation at $16000 \mathrm{rcf}$ at $50{ }^{\circ} \mathrm{C}$, a temperature that is above the $T_{\mathrm{t}}$ of the ELP. The resulting pellet was resuspended in $900 \mu \mathrm{L}$ of conjugation buffer $(0.1$ $\mathrm{M} \mathrm{NaPO}_{4}, 3 \mathrm{mM}$ tris(2-carboxyethyl)phosphine hydrochloride (Thermo Scientific, Waltham, MA)) at room temperature. One milligram of AlexaFluor488-C5 maleimide (Invitrogen, Carlsbad, CA) was then dissolved in $100 \mu \mathrm{L}$ of anhydrous dimethyl sulfoxide (Sigma-Aldrich, St. Louis, MO), immediately mixed with ELP/ binding buffer solution, and continuously rotated at room temperature. The reaction was quenched after $2 \mathrm{~h}$, and excess fluorophore was removed by one round of ITC and desalting via a PD-10 desalting column (GE Healthcare, Waukesha, WI). The ELP-Alexa488 was concentrated to $1 \mathrm{~mL}$ total using the aforementioned ITC method and stored at $-20^{\circ} \mathrm{C}$.

Dynamic Light Scattering. One hundred microliters of $10 \mu \mathrm{M}$ ELP in PBS was filtered using a $0.02 \mu \mathrm{m}$ syringe filter (GE Healthcare), and $35 \mu \mathrm{L}$ of the filtered solution was added to each well of a 384-well plate (Corning, Corning, NY). Small drops of mineral oil were added to the top of each well to prevent evaporation. The wells in the plate were analyzed using a thermally controlled dynamic light scattering Wyatt Plate Reader (Wyatt Technology, Santa Barbara, CA). Ten acquisitions were obtained for each well in $1{ }^{\circ} \mathrm{C}$ increments from 20 to $45^{\circ} \mathrm{C}$. The resulting data were fit using a Rayleigh sphere model and either a regularization or $\mathrm{Cu}$ mulant algorithm based on the sum-of-squares value. Populations comprising less than $2 \%$ of the total mass were excluded from the analysis. These data were used to directly determine the average hydrodynamic ratio $\left(R_{\mathrm{h}}\right)$ and molecular weight $(\mathrm{MW})$ of the particles in solution. The number of unimers per micelle, coordination number, was estimated by

$$
Z=\frac{\mathrm{MW}_{\text {micelle }}}{\mathrm{MW}_{\text {unimer }}}
$$

and ligand density was estimated by

$$
\rho_{\text {ligand }}=\frac{Z}{4 \pi R_{\mathrm{h}}}
$$

The critical micelle temperature (CMT) for each construct was defined as the first temperature where $R_{\mathrm{h}}$ is significantly greater than the average unimer $R_{\mathrm{h}}$.

Thermal Characterization in Serum. The phase transition behavior of each ELP was characterized in serum by monitoring the absorbance of a $10 \mu \mathrm{M}$ ELP in fetal bovine serum (Sigma-Aldrich) at $350 \mathrm{~nm}$ as a function of temperature $\left(1{ }^{\circ} \mathrm{C} / \mathrm{min}\right)$ on a UV - vis spectrophotometer equipped with a multicell thermoelectric temperature controller (Cary 300 Bio; Varian, Inc., Cary, NC). For $E_{E L} P_{B C}$, the CMT was defined as the temperature at which the optical density (OD) first increased from baseline.

Cell Culture. Both wild-type human leukemia K562 cells, K562 $\left(\alpha_{v} \beta_{3}{ }^{-}\right)$, and a stable variant transformed with the gene encod- ing $\alpha_{v} \beta_{3}$ integrin, $K 562 / \alpha_{v} \beta_{3}\left(\alpha_{v} \beta_{3}{ }^{+}\right)$, were a generous gift from Dr. S. Blystone at Upstate Medical University. ${ }^{23}$ Both cell lines were maintained in Iscove's modified Dulbecco's medium (IMDM) (Invitrogen) or RPMI 1640 supplemented with 10\% FBS, $1 \%$ penicillin/streptomycin, and $2 \mathrm{mM} \mathrm{L-glutamine}$ and maintained at $37{ }^{\circ} \mathrm{C}$ and $5 \% \mathrm{CO}_{2} . \mathrm{K} 562-\alpha_{v} \beta_{3}$ media also contained 500 $\mu \mathrm{g} / \mathrm{mL}$ of $\mathrm{G} 418$ (Invitrogen). Flasks were started from frozen cell stocks. Cells were split once every $48 \mathrm{~h}$.

Receptor Expression. A total of $500000 \mathrm{~K} 562$ or $\mathrm{K} 562 / \alpha_{v} \beta_{3}$ cells were plated in 6-well plates and allowed to incubate overnight. Cells were visually inspected, rinsed twice, concentrated to 500 $\mu \mathrm{L}$ via centrifugation, and added to a $1.5 \mathrm{~mL}$ centrifuge tube. Three micrograms of LM609 anti- $\alpha_{v} \beta_{3}$ Ab conjugated to AF488 (Millipore, Billerica, MA) was added to each tube, and cells were incubated at room temperature for $1 \mathrm{~h}$. Cells were then rinsed three times and analyzed by flow cytometry $(n=3)$.

Cell Uptake/Binding. A total of $500000 \mathrm{~K} 562$ or K562/ $\alpha_{v} \beta_{3}$ cells were plated in 6-well plates and allowed to incubate overnight. Cells were visually inspected, rinsed twice, and resuspended in $500 \mu \mathrm{L}$ of a $10 \mu \mathrm{M}$ ELP-AF488 cell suspension (HBSS, $1 \mathrm{mM}$ $\mathrm{CaCl}_{2}$ ). Each sample was rotated at either room temperature or $40{ }^{\circ} \mathrm{C}$ in normal atmosphere for $1 \mathrm{~h}$ and rinsed in binding buffer three times. Cells for flow cytometry analysis were fixed in $4 \%$ PFA for 15 min (Alfa Aesar, Ward Hill, MA) and stored at $4{ }^{\circ} \mathrm{C}$ $(n=3)$. Cells for confocal analysis were immediately mounted on slides and imaged using confocal microscopy.

Flow Cytometry Analysis. Fixed cell samples were analyzed using a LSRII Flow Cytometer (BD Biosciences, San Jose, CA). All samples of fixed cells were analyzed within $18 \mathrm{~h}$ of fixation. Viable cells were gated using the forward scatter and side scatter plots of an unstained control sample. A minimum of 10000 live cells was analyzed per sample. For Ab characterization, cells with intensity 2 standard deviations (SD) over the median intensity of unlabeled control cells were defined as receptor-positive. For binding/uptake experiments, heated cells with intensity $2 \mathrm{SD}$ over the intensity of unheated cells were defined as ELP-positive. Fold increase in median fluorescence intensity was obtained by dividing the corrected median fluorescence intensity of the AlexaFluor + region of the heated sample by the corrected median fluorescence of the unheated sample with otherwise identical conditions.

Confocal Imaging. Five microliters of unfixed cell sample was mixed with a small volume of Fluoromount-G (Electron Microscopy Sciences, Hatfield, PA) and placed on a glass slide. Samples were then mounted and sealed. Slides were then immediately imaged at $5 \times$ and $20 \times$ using an LSM5 Pascal confocal microscope (Zeiss, Oberkotchen, Germany) with two channels for differential interference contrast (DIC) and AlexaFluor488. All images were obtained within $2 \mathrm{~h}$ of slide mounting. Confocal images were not used for quantitative analysis.

Acknowledgment. We thank Jonathan McDaniel for the TOC graphic. This research was supported by the NIH though a grant (R01 EB-007205) to A.C. and by a NSF IGERT fellowship to A.S. (DGE-0221632, Pl: Clark).

Supporting Information Available: Experimental procedures and additional figures. This material is available free of charge via the Internet at http://pubs.acs.org.

\section{REFERENCES AND NOTES}

1. Ehrlich, P. Collected Studies on Immunity; John Wiley \& Sons: New York, 1906.

2. Adams, G. P.; Schier, R.; McCall, A. M.; Simmons, H. H.; Horak, E. M.; Alpaugh, R. K.; Marks, J. D.; Weiner, L. M. High Affinity Restricts the Localization and Tumor Penetration of Single-Chain Fv Antibody Molecules. Cancer Res. 2001, 4750-4755.

3. Caplan, M. R.; Rosca, E. V. Targeting Drugs to Combinations of Receptors: A Modeling Analysis of Potential Specificity. Ann. Biomed. Eng. 2005, 1113-1124.

4. Mauriz, J. L.; Gonzalez-Gallego, J. Antiangiogenic Drugs: Current Knowledge and New Approaches to Cancer Therapy. J. Pharm. Sci. 2008, 4129-4154. 
5. Mammen, M.; Choi, S. K.; Whitesides, G. M. Polyvalent Interactions in Biological Systems: Implications for Design and Use of Multivalent Ligands and Inhibitors. Angew. Chem., Int. Ed. 1998, 2754-2974.

6. Peer, D.; Karp, J. M.; Hong, S.; Farokhzad, O. C.; Margalit, R.; Langer, R. Nanocarriers as an Emerging Platform for Cancer Therapy. Nat. Nanotechnol. 2007, 751-760.

7. Meyer, D. E.; Chilkoti, A. Genetically Encoded Synthesis of Protein-Based Polymers with Precisely Specified Molecular Weight and Sequence by Recursive Directional Ligation: Examples from the Elastin-like Polypeptide System. Biomacromolecules 2002, 357-367.

8. Urry, D. W. Free Energy Transduction in Polypeptides and Proteins Based on Inverse Temperature Transitions. Prog. Biophys. Mol. Biol. 1992, 23-57.

9. Urry, D. W. Physical Chemistry of Biological Free Energy Transduction as Demonstrated by Elastic Protein-Based Polymerst. J. Phys. Chem. B 1997, 11007-11028.

10. Dreher, M. R.; Simnick, A. J.; Fischer, K.; Smith, R. J.; Patel, A.; Schmidt, M.; Chilkoti, A. Temperature Triggered SelfAssembly of Polypeptides into Multivalent Spherical Micelles. J. Am. Chem. Soc. 2008, 687-694.

11. Lee, T. A. T.; Cooper, A.; Apkarian, R. P.; Conticello, V. P. Thermo-Reversible Self-Assembly of Nanoparticles Derived from Elastin-Mimetic Polypeptides. Adv. Mater. 2000 1105-1110.

12. Meyer, D. E.; Chilkoti, A. Purification of Recombinant Proteins by Fusion with Thermally-Responsive Polypeptides. Nat. Biotechnol. 1999, 1112-1115.

13. Koivunen, E.; Gay, D. A.; Ruoslahti, E. Selection of Peptides Binding to the A5 $\beta 1$ Integrin from Phage Display Library. J. Biol. Chem. 1993, 20205-20210.

14. Gladson, C. L.; Cheresh, D. A. Glioblastoma Expression of Vitronectin and the $\alpha_{\mathrm{v}} \beta_{3}$ Integrin. Adhesion Mechanism for Transformed Glial Cells. J. Clin. Invest. 1991, 1924-1932.

15. Max, R.; Roland, R. C.; Gerritsen, M.; Peet, T. G.; Nooijen, A.; Goodman, S. L.; Sutter, A.; Keilholz, U.; Ruiter, D. J.; De Waal, R. M. W. Immunohistochemical Analysis of Integrin $\alpha_{v} \beta_{3}$ Expression on Tumor-Associated Vessels of Human Carcinomas. Int. J. Cancer 1997, 320-324.

16. Lindemann, S.; Kramer, B.; Seizer, P.; Gawaz, M. Platelets, Inflammation and Atherosclerosis. J. Thromb. Haemostasis 2007, 203-211.

17. Winter, P. M.; Morawski, A. M.; Caruthers, S. D.; Fuhrhop, R. W.; Zhang, H.; Williams, T. A.; Allen, J. S.; Lacy, E. K.; Robertson, J. D.; Lanza, G. M.; Wickline, S. A. Molecular Imaging of Angiogenesis in Early-Stage Atherosclerosis with $\alpha_{v} \beta_{3}$-Integrin-Targeted Nanoparticles. Circulation 2003, 2270-2274.

18. Vagnucci, A. H.; Li, W. W. Alzheimer's Disease and Angiogenesis. The Lancet 2003, 605-608.

19. Ruoslahti, E. Rgd and Other Recognition Sequences for Integrins. Annu. Rev. Cell Dev. Biol. 1996, 697-715.

20. Giancotti, F. G.; Ruoslahti, E. Integrin Signaling. Science 1999, 1028-1033.

21. Dreher, M. R.; Raucher, D.; Balu, N.; Michael Colvin, O.; Ludeman, S. M.; Chilkoti, A. Evaluation of an Elastin-like Polypeptide-Doxorubicin Conjugate for Cancer Therapy. J. Controlled Release 2003, 31-43.

22. Maheshwari, G.; Brown, G.; Lauffenburger, D. A.; Wells, A.; Griffith, L. G. Cell Adhesion and Motility Depend on Nanoscale Rgd Clustering. J. Cell. Sci. 2000, 1677-1686.

23. Blystone, S. D.; Graham, I. L.; Lindberg, F. P.; Brown, E. J. Integrin $\alpha_{v} \beta_{3}$ Differentially Regulates Adhesive and Phagocytic Functions of the Fibronectin Receptor $\alpha_{5} \beta_{1}$. J. Cell Biol. 1994, 1129-1137.

24. Raucher, D.; Chilkoti, A. Enhanced Uptake of a Thermally Responsive Polypeptide by Tumor Cells in Response to Its Hyperthermia-Mediated Phase Transition. Cancer Res. 2001, 7163-7170.

25. Maheshwari, G.; Brown, G.; Lauffenburger, D. A.; Wells, A.; Griffith, L. G. Cell Adhesion and Motility Depend on Nanoscale Rgd Clustering. J. Cell Sci. 2000, 1677-1686.
26. Urano, M. Invited Review: For the Clinical Application of Thermochemotherapy Given at Mild Temperatures. Int. J. Hypertherm. 1999, 79-107. 